\title{
Phyllosoma de Jasus frontalis (Decapoda: Palinuridae) en la costa continental de Chile
}

\author{
Jasus frontalis phyllosoma (Decapoda: Palinuridae) in the continental coast of Chile
}

Armando Mujica1, Maria L. Nava' ${ }^{1}$ y Eduardo Flores ${ }^{1}$

${ }^{1}$ Facultad de Ciencias del Mar, Universidad Católica del Norte, Casilla 117, Coquimbo, Chile. amujica@ucn.cl

\begin{abstract}
The presence of a Palinuridae phyllosoma larvae near Salado Bay (270’S) north-central coast of Chile, is reported. The identification is discussed and the possibility of this larvae being Projasus bahamondei, which is the only species from the family that is described for the area, is discarded. The shape, size and position of the cephalic, pereionic, abdominal and telson appendices concords with descriptions of advanced stage J asus frontalis larvae. Moreover, the specimen described here has five pairs of biramous pereiopods with setose exopods. In accordance with these observations and published data, it is proposed that this phyllosoma corresponds to a stage XII or XIII of $\mathrm{J}$. frontalis larva.
\end{abstract}

Key words: Rock lobster, larvae, Sudamerican Pacific coast

\section{INTRODUCCIÓN}

Para las costas de Chile, se han descrito tres especies de la familia Palinuridae, Jasus frontalis (Milne Edwards, 1837), Panulirus pascuensis Reed, 1954 y Projasus bahamondei George, 1976, distribuidas principalmente en las costas de las islas oceánicas chilenas (Rivera \& Mujica 2004). Sólo P. bahamondei se ha descrito para las costas continentales del centro y norte de Chile (Báez \& Ruíz 2000). Retamal (2000) la reporta desde Huasco (2828'S) a Constitución (35²0’S), en el archipiélago Juan Fernández e islas Desventuradas, en profundidades comprendidas entre 175 y $550 \mathrm{~m}$.

Los estados larvales de estas especies, phyllosomas y puerulus, son conocidos parcialmente (Wehrtmann \& Báez 1997). Báez (1973) y Dupre \& Guisado (1996), describen algunos estadios larvales de $J$. frontalis, mientras que Báez \& Ruiz (2000) describen el puerulus de Projasus bahamondei. Báez (1973), tentativamente asigna a $P$. bahamondei el phyllosoma que denomina "phyllosoma X", aunque Webber \& Booth (1988), señalan que éste debería pertenecer a otra especie. Rivera \& Mujica (2004), discuten la controversia planteada en la identificación de esta larva, señalando que su morfología es más próxima a la del género Projasus que a la de Jasus o Panulirus.

El presente trabajo reporta el hallazgo de un phyllosoma de Jasus frontalis encontrado en la costa continental de la zona norte de Chile y se discute su identificación.

\section{Material y MÉTODOS}

En el crucero de prospección del proyecto "Evaluación hidroacústica del reclutamiento de anchoveta en la III y IV Regiones, año 2008", financiado por el Fondo de Investigación Pesquera de Chile, FIP 2007-03, efectuado en febrero del 2008 a bordo del B/I Abate Molina del Instituto de Fomento Pesquero (IFOP), se tomaron muestras de zooplancton en 100 estaciones distribuidas

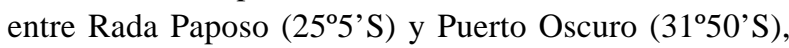
mediante el uso de redes bongo (59 cm de diámetro, 300 $\mu \mathrm{m}$ de abertura de malla y provistas de flujómetro), que se arrastraron en forma oblicua entre la superficie y $70 \mathrm{~m}$ de profundidad (Fig. 1).

Las muestras se preservaron en solución de formalina al $4 \%$ disuelta en agua de mar, para el posterior análisis en laboratorio. El número de zooplancteres capturados se estandarizó en $1000 \mathrm{~m}^{3}$ de agua de mar filtrada.

\section{RESULTADOS Y DISCUSIÓN}

El 17 de febrero de 2008 en la estación 43 nocturna (00:42 h), ubicada a 6 millas náuticas (mn) de la costa, al oeste de Punta Cachos, Bahía Salado (27\%40’S; $71^{\circ} 09^{\prime}$ W), se capturó la larva phyllosoma en estado avanzado de desarrollo.

La estación en la que se capturó el phyllosoma, se encuentra dentro del rango de distribución de Projasus bahamondei (Retamal 2000), que es la única especie Palinura descrita para estas aguas. Sin embargo, las 

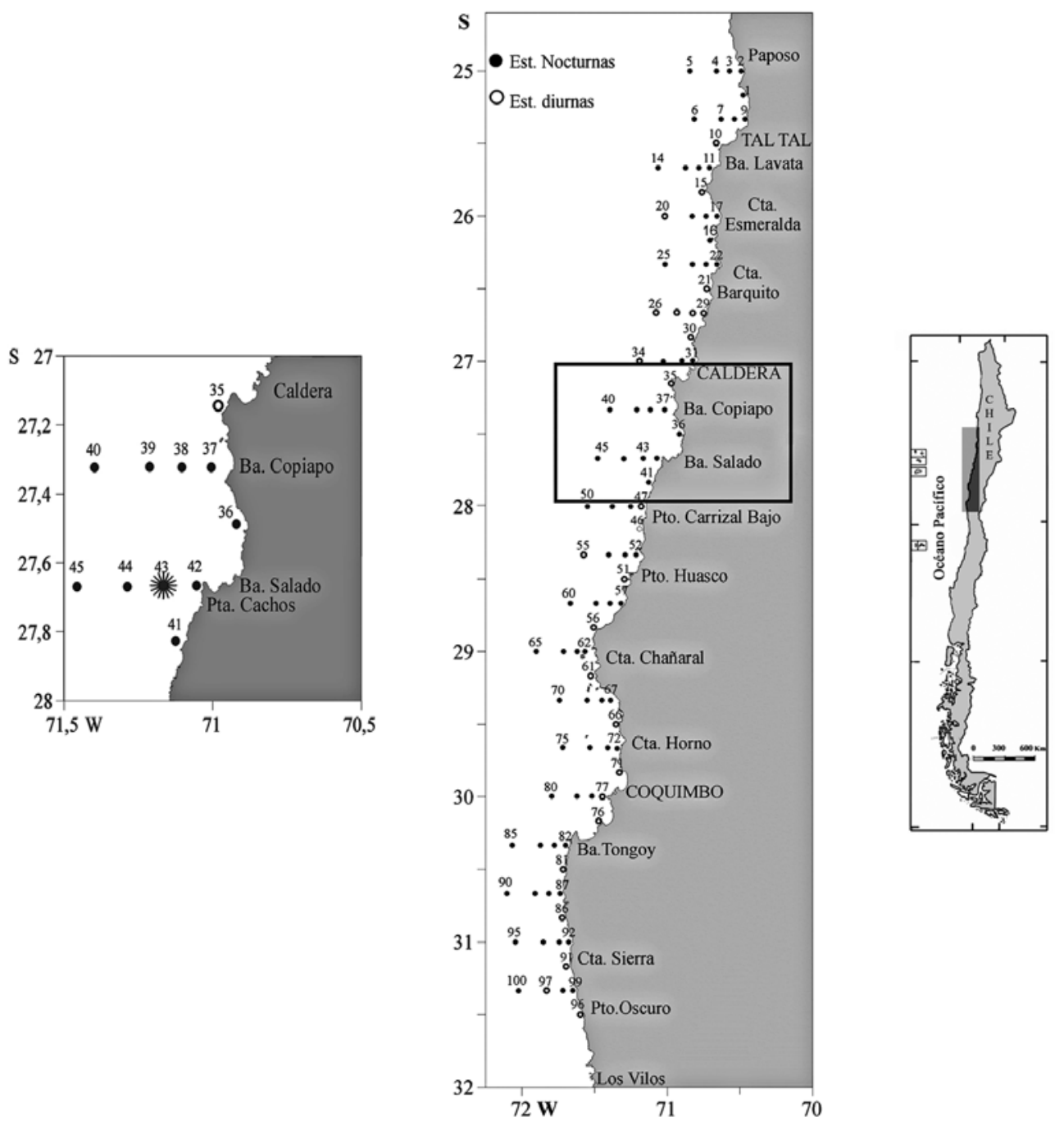

Figura 1. Ubicación de estaciones de muestreo de larvas de decápodos en la costa norte-centro de Chile / Location of sampling station for larval decapods from the northern-central coast of Chile

características morfológicas del ejemplar capturado, difieren considerablemente de la descripción del "phyllosoma X" (Báez 1973), que pertenecería a $P$. bahamondei (Rivera \& Mujica 2004).

La forma semicircular y tamaño del cefalon (19 mm de largo y $27 \mathrm{~mm}$ de ancho), el emplazamiento de los pedúnculos oculares, anténulas y antenas, corresponden con la descripción de los estadios avanzados de Jasus frontalis (Báez 1973), no obstante, la longitud de las antenas sobrepasa la proporción señalada por este autor. La morfología general del pereión, abdomen y telson, corresponden con la descripción de los phyllosomas más desarrollados descritos para la especie, así como también sus apéndices en general (Fig. 2).

El ejemplar descrito tiene los cinco pares de pereiópodos birramosos con exopoditos setosos (Fig. 3), similar a los cuatro primeros pares descritos por Báez (1973) en phyllosomas XI y XIIIA. Las descripciones de este autor están basadas en ejemplares con deterioro de sus apéndices y el quinto par de pereiópodo seccionados, sin descripción de su estructura birramosa y la presencia de setas plumosas en el exopodito, lo cual difiere del phyllosoma VIII, que lo describe como unirramoso. 


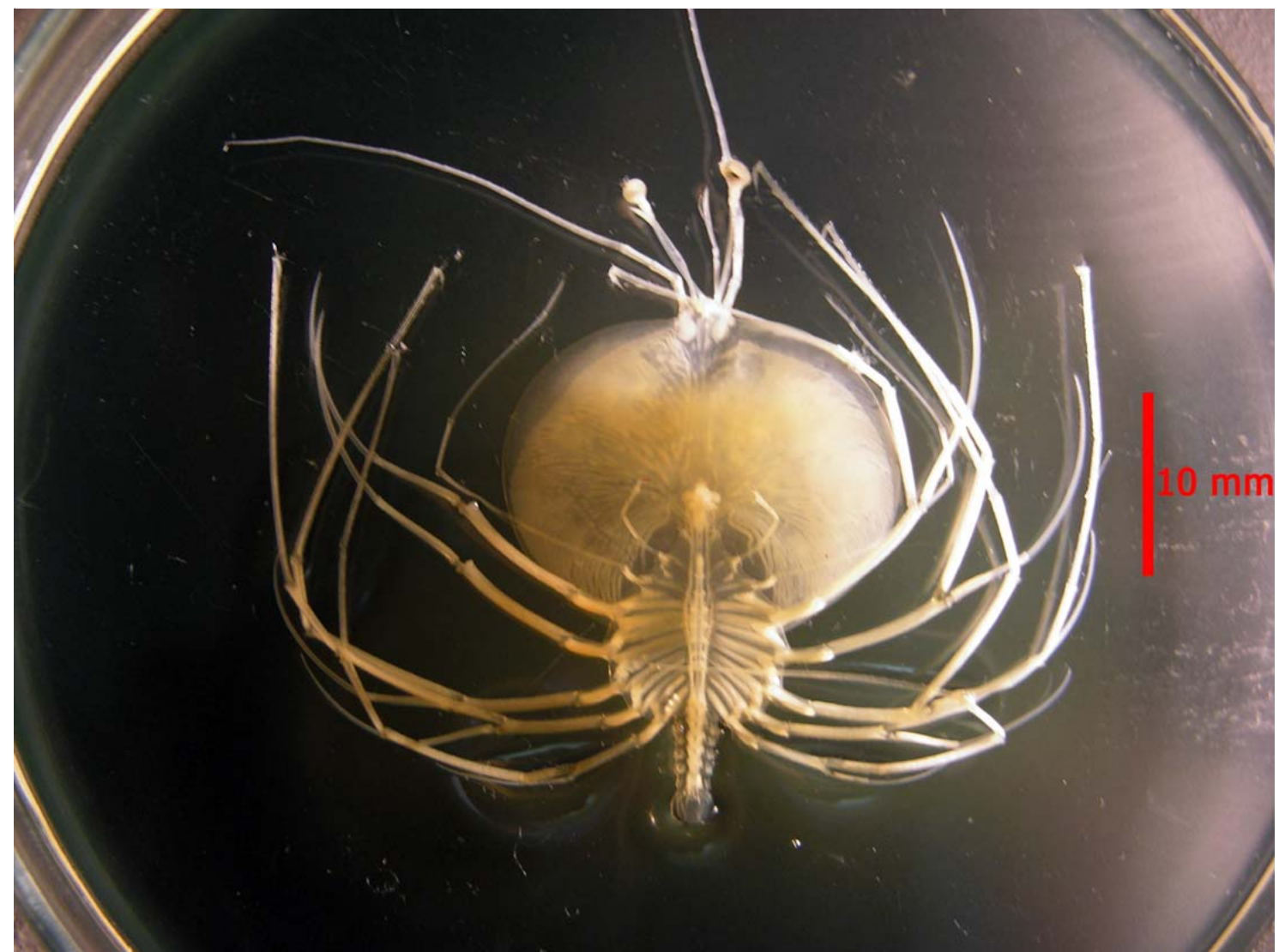

Figura 2. Vista ventral de phyllosoma XII o XIII de Jasus frontalis / Ventral view of phyllosoma XII or XIII of J asus frontalis

De acuerdo a lo anterior y a los antecedentes disponibles (Gurney 1936, Johnson 1971, Báez 1973, 1983, Ito \& Lucas 1990, Dupré \& Guisado 1996, Kittaka et al. 1997, Coutures 2001, Rivera \& Mujica 2004), se postula que el phyllosoma capturado frente a bahía Salado, corresponde a un estado XII o XIII de Jasus frontalis, que difiere de las descripciones de otros estadios, por la longitud de las antenas y el borde posterior de cefalon, que no es levemente aguzado.

La distribución geográfica de Jasus frontalis en Chile, se restringe a las costas insulares del archipiélago de Juan Fernández, (islas Robinson Crusoe, Santa Clara y Alejandro Selkirk) y de las islas Desventuradas (San Félix y San Ambrosio) (Retamal 2000). Las primeras islas se encuentran a $430 \mathrm{mn}$ al oeste del litoral central de Chile,

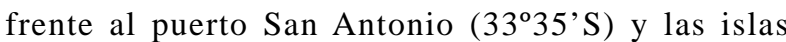

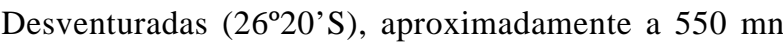
frente a la costa norte de Chile, comprendida entre Caldera y Taltal, por lo que la presencia de phyllosomas de la especie frente a las costas continentales de Chile, constituye un hallazgo sin precedente.

La presencia de este phyllosoma a cientos de millas náuticas de los posibles lugares de origen, se ha relacionado con el prolongado desarrollo larval y el transporte advectivo a la que están sujetas las larvas de langostas, considerando lugares tan aislados como las islas oceánicas del océano Pacífico Suroriental (Báez 1973, Lutjeharms \& Heydorn 1981a, b, Booth 1997). La distancia que pueden recorrer las larvas debido a la advección depende del tiempo que permanecen en el plancton, por lo que especies de largo desarrollo larval, podrían recorrer grandes distancias, lo que explicaría la presencia esporádica de ejemplares en sitios donde no es característica la presencia de los adultos (Booth \& Ovenden 2000). 

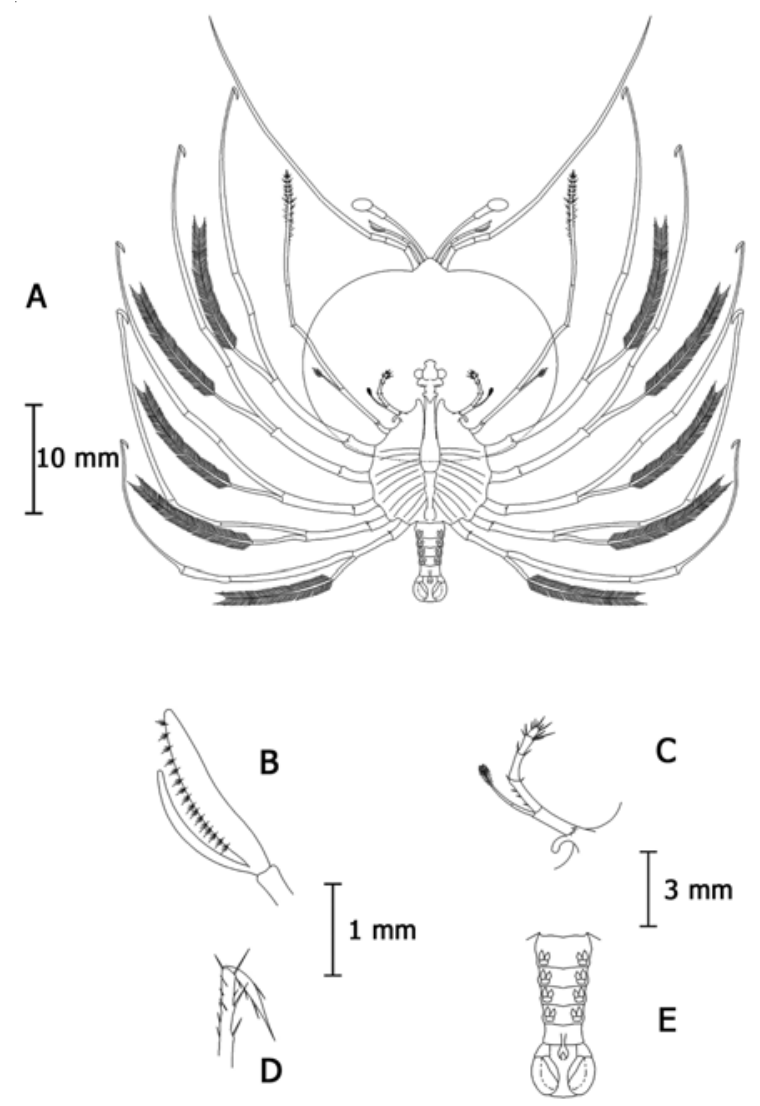

Figura 3. A) Vista ventral de phyllosoma XII o XIII de Jasus frontalis, B) antenula, C) segundo maxilípedo, D) dáctilo de periópodos, E) abdomen, urópodos y telson / A) Ventral view of phyllosoma XII or XIII of J asus frontalis, B) antennule, C) second maxilliped, D) pereiopod dactylus, E) abdomen, uropod and telson

El desarrollo larval de las especies de la familia Palinuridae, está compuesto por 11 a 17 estadios, lo que implica hasta 11 meses de vida planctónica (Atkinson \& Bouster 1982, Ito \& Lucas 1990, Kittaka et al. 1997, Rivera \& Mujica 2004). Para Jasus frontalis se ha postulado una duración de 112 a 378 días entre los estados de naupliosoma y puerulus (Dupré \& Guisado 1996).

Por otra parte, Rimmer \& Phillips (1979), determinaron que los phyllosoma intermedios y avanzados de Panulirus cygnus alcanzan velocidades de 16 a $20 \mathrm{~m} \mathrm{~h}^{-1}$, mientras que Dupré \& Guisado (1996), establecen que el primer phyllosoma de Jasus frontalis, desarrolla velocidades comprendidas entre 0,54 y $0,75 \mathrm{~m} \mathrm{~min}^{-1}$ en laboratorio (32-45 $\left.\mathrm{m} \mathrm{h}^{-1}\right)$.
Adicionalmente, la migración vertical de las larvas de Panulirus cyngnus (Rimmer \& Phillips 1979) y otras especies (Rivera \& Mujica 2004), explica las mayores concentraciones de phyllosomas en pescas superficiales nocturnas y a más profundidad durante horas de luz, que se relaciona con el comportamiento larval favoreciendo la dispersión y/o retención de éstas. Al respecto, Phillip et al. (1978) señalaron que las primeras phyllosomas serían transportadas costa afuera por las corrientes superficiales, mientras que los estadios intermedios y avanzados estarían regidos por la circulación de las aguas subsuperficiales, con el transporte hacia las áreas cercanas de las islas y montes submarinos para su reclutamiento.

Por otro lado, las islas Desventuradas están influidas la mayor parte del año por aguas ecuatoriales subsuperficiales de la contracorriente Perú-Chile, las que en verano austral alcanzan el archipiélago de Juan Fernández, que el resto del año, está influenciado por aguas subantárticas, que a su vez, alcanzan las islas Desventuradas en invierno austral (Bahamonde 1987). Fuenzalida et al. (2007) y Schneider et al. (2007), discuten la existencia de numerosos flujos y contra flujos en direcciones norte y sur respectivamente, que se relacionan con las fronteras que separan las aguas frías y cálidas que se desplazan en sentidos opuestos. Estas características hidrográficas son determinantes en la dispersión o retención de las larvas phyllosomas de los géneros Jasus, Scyllarus y Projasus (phyllosoma X), entre las islas Desventuradas y el archipiélago de Juan Fernández (Rivera \& Mujica 2004), estos autores, señalaron que un factor determinante en la dispersión de los géneros antes mencionados, es la topografía de fondo que generaría una barrera para el asentamiento de las larvas que completan su desarrollo, lo cual mantendría el proceso de reclutamiento dentro de los cordones de montes submarinos que conforman las islas Desventuradas y el archipiélago de Juan Fernández.

\section{Agradecimientos}

Los autores agradecen a la tripulación del B/I Abate Molina y profesionales del IFOP que cooperaron en la toma de muestras planctónicas. Al Sr. Felipe Mujica, quien diagramó las figuras del phyllosoma descrito.

\section{LITERATURA CITADA}

Atkinson J \& N Boustead. 1982. The complete larval development of the scyllarii lobster Ibacus alticrenatus Bate, 1888 in New Zeland waters. Crustaceana 42(3): 275287. 
Báez P. 1973. Larvas Phyllosoma del Pacifico sur oriental (Crustacea, Macrura, Scyllaridea). Revista de Biología Marina 15(1): 115-130.

Báez P. 1983. Larvas phyllosomas y puerulus de la langosta verde Panulirus gracilis Strets 1871, procedentes de la expedición Costa Rica, 1973 (Crustacea: Decapoda: Palinuridae). Revista de Biología Marina 19(1): 79-111.

Báez P \& R Ruiz. 2000. Puerulus y postpuerulus de Projasus bahamondei George, 1976 (Crustacea, Decapoda, Palinuridae). Investigaciones Marinas 28: 15-25.

Bahamonde N. 1987. San Félix y San Ambrosio, las islas llamadas Desventuradas. En: Castilla JC (ed). Islas oceánicas chilenas: Conocimiento científico y necesidades de investigaciones, pp. 85-100. Ediciones Universidad Católica de Chile, Santiago.

Booth JD. 1997. Long-distance movements in Jasus spp. and their role in larval recruitment. Bulletin of Marine Science 61(1): 111-128.

Bood JD \& JR Ovenden. 2000. Distribution of Jasus spp. (Decapoda: Palinuridae) phyllosomas in southern waters: implications for larval recruitment. Marine Ecology Progress Series 200: 241-255.

Coutures E. 2001. On the first phyllosoma stage of Parribacus caledonicus Holthuis, 1960, Scyllarides squamosus $(\mathrm{H}$. Milne-Edwards, 1837) and Arctides regalis Holthuis, 1963 (Crustacea, Decapoda, Scyllaridae) from New Caledonia. Journal of Plankton Research 23(7): 745-751.

Dupré E \& C Guisado. 1996. Identificación de los primeros estados de phylosoma de la langosta de Juan Fernández (Jasus frontalis) mantenidos en laboratorio. Investigaciones Marinas 24: 39-50.

Fuenzalida R, W Schneider, JL Blanco, J Garcés-Vargas \& L Bravo. 2007. Sistema de corrientes Chile-Perú y masas de agua entre Caldera e Isla de Pascua. Ciencia y Tecnología del Mar 30(2): 5-16.

Gurney R. 1936. Larvae of decapod crustacea. Part III, Phyllosoma. Discovery Report 12: 400-440.

Ito M \& J Lucas. 1990. The complete larval development of the scillrid lobster, Scyllarus demani Holthuis, 1946 (Decapoda, Scyllaridae), in the laboratory. Crustaceana 58(2): 144-167.
Johnson M. 1971. The palinurid and scyllarid lobster larvae of the tropical eastern Pacific and their distribution as related to the prevailing hydrography. Bulletin of the Scripps Institution of Oceanography 19: 1-36.

Kittaka J, K Ono \& J Booth. 1997. Complete development of the green rock lobster, Jasus verreauxi from egg to juvenile. Bulletin of Marine Science 61(1): 57-71.

Lutjeharms JR \& EF Heydorn. 1981a. The rock-lobster Jasus tristani on Vema Seamount: drifting buoys suggest a possible recruiting mechanism. Deep Sea Research 28A(6): 631-636.

Lutjeharms JR \& EF Heydorn. 1981b. Recruitment of rock lobster on Vema Seamount from the island of Tristan da Cunha. Deep Sea Research 28A(10): 1237.

Phillip B, D Rimmer \& D Reid. 1978. Ecological investigations of the late stage phyllosoma and puerulus larvae of the western rock lobster Panulirus longipes cygnus. Marine Biology 45: 347-357.

Retamal M. 2000. Decápodos de Chile. 1995. World Biodiversity Database CD-ROM Series. ETI-U. de Concepción. Springer-Verlag.[CD-ROM]

Richards W \& J Goulet. 1976. An operational surface drift model used for studying larval lobster recruitment and dispersal. FAO Fisheries Report 200: 363-374.

Rimmer D \& B Phillips. 1979. Diurnal migration and vertical distribution of phyllosoma larvae of the western rock lobster Panulirus cygnus. Marine Biology 54: 109-124.

Rivera J \& A Mujica. 2004. Larvas phyllosoma (Decapoda, Palinuridae y Scyllaridae) de las islas oceánicas chilenas. Investigaciones Marinas 32(2): 99-111.

Schneider W, R Fuenzalida, R Nuñez, R Bravo \& D Figueroa. 2007. Discusión del Sistema de la Corriente de Humboldt y masas de agua en la zona norte y centro de Chile. Ciencia y Tecnología del Mar 30(1): 21-36.

Webber W \& J Booth. 1988. Projasus parkeri (Stebbing, 1902) (Crustacea, Decapoda, Palinuridae) in New Zealand and description of a Projasus puerulus from Australia. National Museum New Zealand Records 3(8): 81-92.

Wehrtmann I \& P Báez. 1997. Larvas y estadíos tempranos de desarrollo de crustáceos decápodos de Chile. Descripciones publicadas. Investigaciones Marinas 25: 263276.

Recibido el 14 de agosto de 2009 y aceptado el 14 de abril de 2010 